\title{
A Simple and Efficient Boolean Solver for Constraint Logic Programming
}

\author{
Philippe Codognet and Daniel Diaz \\ INRIA-Rocquencourt \\ Domaine de Voluceau BP 105 \\ 78153 Le Chesnay Cedex \\ FRANCE \\ $\{$ Philippe.Codognet, Daniel.Diaz\}@inria.fr
}

\begin{abstract}
We study in this paper the use of consistency techniques and local propagation methods, originally developped for constraints over finite domains, for solving boolean constraints in Constraint Logic Programming (CLP). We present a boolean CLP language $c l p(B / F D)$ built upon a CLP language over finite domains $c l p(F D)$ which uses a propagation-based constraint solver. It is based on a single primitive constraint which allows the boolean solver to be encoded at a low-level. The boolean solver obtained in this way is both very simple and very efficient: on average it is eight times faster than the CHIP propagation-based boolean solver, i.e. nearly an order of magnitude faster, and infinitely better than the CHIP boolean unification solver. It also performs on average several times faster than special-purpose stand-alone boolean solvers. We further present several simplifications of the above approach, leading to the design of a very simple and compact dedicated boolean solver. This solver can be implemented in a WAM-based logical engine with a minimal extension limited to four new abstract instructions. This $\operatorname{clp}(B)$ system provides a further factor two speedup w.r.t. clp(B/FD).
\end{abstract}

\section{Introduction}

Constraint Logic Programming combines both the declarativity of Logic Programming and the ability to reason and compute with partial information (constraints) on specific domains, thus opening up a wide range of applications. Among the usual domains found in CLP, the most widely investigated are certainly finite domains, $\mathrm{real} /$ rationals with arithmetic constraints, and booleans. This is exemplified by the three main CLP languages: CHIP 31, which proposes finite domains, rational and booleans, PrologIII [11] which includes rationals, booleans and lists, and $\operatorname{CLP}(\mathcal{R})[18$ which handles contraints over reals. Whereas most researchers agree on the basic algorithms used in the constraint solvers for reals/rationals (simplex and gaussian elimination) and finite domains (local propagation and consistency techniques), there are many different approaches proposed for boolean constraint solving. Some of these solvers provide special-purpose boolean solvers while others have been integrated inside a CLP framework. However, different algorithms have different performances, and it is hard to know if, for some particular application, any specific solver will be able to solve it in practise. Obviously, the well-known NP-completeness of the satisfiability of boolean formulas shows that we are tackling 
a difficult problem here.

Over recent years, local propagation methods, developed in the CHIP language for finite domain constraints [31, have gained a great success for many applications, including real-life industrial problems. They stem from consistency techniques introduced in AI for Constraint Satisfaction Problems (CSP) [19. Such techniques have also been used in CHIP to solve boolean constraints with some success; in fact to such an extent that it has become the standard tool in the commercial version of CHIP. This method performs better than the original boolean unification algorithm for nearly all problems and is competitive with special-purpose boolean solvers. Thus, the basic idea is that an efficient boolean solver can be derived from a finite domain constraint solver for free.

It was therefore quite natural to investigate such a possiblity with our CLP system clp(FD), which handles finite domains constraints similar to that of CHIP, but being nevertheless about four times faster on average [13, 10. clp(FD) is based on the so-called "glass-box" approach proposed by [32, as opposed to the "blackbox" approach of the CHIP solver for instance. The basic idea is to have a single constraint $X$ in $r$, where $r$ denotes a range (e.g. $t_{1} . . t_{2}$ ). More complex constraints such as linear equations and inequations are then defined in terms of this primitive constraint. The $X$ in $r$ constraint can be seen as embedding the core propagation mechanism for constraint solving over finite domains, and can be seen as an abstract machine for propagation-based constraint solving.

We can therefore directly encode a boolean solver at a low-level with this basic mechanism, and decompose boolean constraints such as and, or, and not in $X$ in $r$ expressions. In this way, we obtain a boolean solver which is obviously more efficient than the encoding of booleans with arithmetic constraints or with the less adequate primitives of CHIP. Worth noticing is that this boolean extension, called clp (B/FD), is very simple; the overall solver (coding of boolean constraints in $X$ in $r$ expression) being about ten lines long, the glass-box is very clear indeed... Moreover, this solver is surprisingly very efficient, being eight times faster on average than the CHIP solver (reckoned to be efficient), with peak speedup reaching two orders of magnitude in some cases. $c l p(B / F D)$ is also more efficient than special purpose solvers, such as solvers based on Binary Decision Diagrams (BDDs), enumerative methods or schemes using techniques borrowed from Operational Research. This architecture also has several other advantages, as follows. First, being integrated in a full CLP language, heuristics can be added in the program itself, as opposed to a closed boolean solver with (a finite set of) built-in heuristics. Second, being integrated in a finite domain solver, various extensions such as pseudo-booleans [6] or multi-valued logics [33] can be integrated straightforwardly. Third, being based on a propagation method, searching for a single solution can be done much more quickly if the computation of all solutions is not needed.

Nevertheless, performances can be improved by simplifying the data-structures used in $\operatorname{clp}(\mathrm{FD})$, which are indeed designed for full finite domain constraints. They can be specialized by introducing explicitly a new type and new instructions for boolean variables. It is possible, for instance, to reduce the data-structure representing the domain of a variable and its associated constraints to only two words: one pointing to the chain of constraints to awake when the variable is bound to 0 and the other when it is bound to 1 . Also some other data-structures become useless for boolean variables, and can be simplified. Such a solver is very compact and simple; it is based again on the glass-box approach, and uses only a single low-level constraint, more specialized than the $X$ in $r$ construct, into which boolean constraints such as and, or or not are decomposed. This primitive constraint can be implemented 
into a WAM-based logical engine with a minimal extension : only four new abstract instructions are needed. This dedicated solver, called $\operatorname{clp}(\mathrm{B})$, provides a further factor two speedup w.r.t. clp(B/FD).

The rest of this paper is organized as follows. Section 2 reviews the variety of methods already proposed for solving boolean constraints and presents in particular the use of local propagation and consistency techniques. Section 3 introduces the formalization of the semantics of propagation-based boolean solvers as a particular constraint system. Section 4 then proposes a first implementation on top of the clp(FD) system by using the $X$ in $r$ primitive constraint for decomposing boolean constraints. The performances of this system, called clp(B/FD), are evaluated in section 5, and compared both with the CHIP system and with several other efficient dedicated boolean solvers. In section 6 , a number of simplifications of the previous approach are proposed, leading to the design of a very simple and compact specialized boolean solver, called $\operatorname{clp}(\mathrm{B})$, performances of which are detailed in section 7. A short conclusion and research perspectives end the paper.

\section{A review of Boolean solvers}

Although the problem of satisfiability of a set of boolean formulas is quite old, designing efficient methods is still an active area of research, and there has been a variety of methods proposed over recent years toward this aim. Moreover, it is usually important not only to test for satisfiability but also to actually compute the models (assignments of variables), if any. To do so, several types of methods have been developed, based on very different data-structures and algorithms. Focusing on implemented systems, existing boolean solvers can be roughly classified as follows.

\section{$2.1 \quad$ Resolution-based methods}

The resolution method, which has been proposed for full first-order logic, can obviously be specialized for propositional logic and therefore be used for solving boolean problems. Such a method is based on clausal representation for boolean formulas, each literal representing a boolean variable, i.e. conjunctive normal form. The core of the method will consist in trying to apply resolution steps between clauses containing occurences of the same variable with opposite signs until either the empty clause (inconsistency) is derived, or some desired consequence of the original formulas is derived. SL-resolution is for instance used for solving boolean constraints in the current version of the Prolog-III language [11] 2]. However, the performances of this solver are very poor and limit its use to small problems. Many refinements have been proposed for limiting the potentially huge search space that have to be explored in resolution-based methods, see 24 for a detailed discussion and further references. Another improved resolution-based algorithm, using a relaxation procedure, is described in [15. However, there does not seem to be any general solution which can improve efficiency for a large class of problems.

\subsection{BDD-based methods}

Binary Decision Diagrams (BDD) have recently gained great success as an efficient way to encode boolean functions [7, and it was natural to try to use them in boolean solvers. The basic idea of BDDs is to have a compact representation of the Shanon 
normal form of a boolean formula. A formula is in normal form if it is reduced to a constant ( 0 or 1$)$, or an expression of the form ite $(x, F, G)$, meaning "if $x$ then $F$ else $G$ ", where $F$ and $G$ are in normal form. An if-then-else expression of the form ite $(x, F, G)$ represents the formula $(x \wedge F) \vee(\neg x \wedge G)$. An efficient way to encode and manipulate this normal form is to use a reduced and ordered binary decision diagram, which is represented as a directed binary acyclic graph whose interior nodes are labeled by variables and leaves by constants 0 and 1 . An interior node $x$ with two sons $F$ and $G$ represents an ite expression ite $(x, F, G)$. Assuming a total order on the set of boolean variables, it is possible to construct for any boolean formula a BDD that respects this ordering (i.e. $x<y$ iff there is a path from $x$ to $y$ ) such that common subexpressions are merged. It is possible to achieve in this way a unique normal form. For example, let us consider the formula $F=(x \wedge y) \vee z$ and the ordering $x<y<z$. The corresponding BDD is depicted in figure 1 .

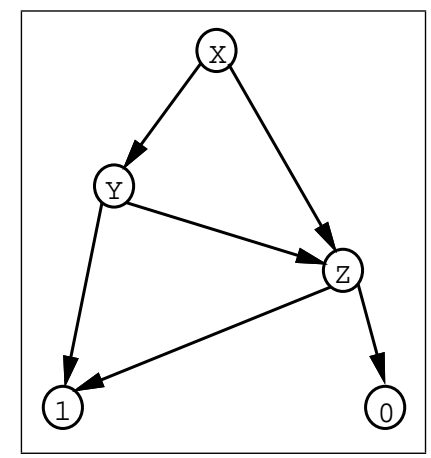

Figure 1: BDD encoding for $(x \wedge y) \vee z$

The size and the form of the BDD are very dependent on the ordering of variables chosen, as a good order will amount to many common subexpressions to be merged while a bad one to none. Therefore, the number of nodes of the BDD can be, depending on the ordering, from linear to exponential w.r.t. the initial number of variables.

Nevertheless, BDD have been used as basic encoding for formulas in many solvers. For instance, the boolean unification 20 solver of CHIP uses such a BDD representation [8] [30]. Other solvers using BDDs include the Adia solver [25, its improved version (second method of [27]) and the Wamcc-Adia combination [14. The Wamcc-Adia system consists of an integration of a BDD-based boolean solver into a Prolog compiler based on the WAM (Warren Abstract Machine), at the abstract intruction level. It performs about four times faster than CHIP's boolean unification [14. Such solvers are efficient for some circuit verification applications, but do not have as good results for less symmetrical problems, e.g. traditional boolean benchmarks, for the size of the BDD during the computation can become very large. It is also very costly to maintain a normal form (the BDD) and to recompute it each time a new constraint is added. Moreover, performances are very dependent of the ordering chosen, and it is not really possible to include in CLP languages complex ordering heuristics, because of the incremental construction of the BDD during program execution. Moreover, these solvers are unable to selectively compute, if desired, a single solution instead of all possible ones. 


\subsection{Enumerative methods}

These methods roughly consist in trying possible assignments by incrementally instanciating variables to 0 or 1 and checking consistency in various sophisticated ways. The seminal algorithm by Davis and Putman falls into this category, although it can also be reformulated in the previous resolution framework. The basic idea is to build, either implicitly or explicitly, a decision tree by instanciating variables and backtracking. Boolean constraints are checked for consistency in sophisticated ways as soon as all their variables become ground. 24] and 21] contain various improvements in consistency checking, and [26] shows how to compute most general unifiers representing all possible models. New methods use a matrix-based clausal form to represent constraints for efficiency reasons, either by bit-vector encoding 21] or with a spare-matrix representation (first method of [27]). They also allow fixed variables to be detected quickly. These solvers can be made quite efficient by introducing various heuristics.

\section{$2.4 \quad 0-1$ integer programming}

A very different method was recently proposed, consisting in encoding constraint satisfaction problems, and in particular boolean problems, as sets of linear inequations over integers such that the satisfiability of the initial problem reduces to an optimisation problem for the solution of the derived set of inequations [17. Established methods from Operational Research, and in particular branch-and-cut methods for 0-1 programming can then be used to perform this computation. The idea is to start from the clausal representation of the boolean problem and to translate each clause in a straightforward way. For instance clauses such as $x_{1} \vee \neg x_{2} \vee x_{3} \vee \neg x_{4}$ will be translated into the linear inequation $x_{1}+\left(1-x_{2}\right)+x_{3}+\left(1-x_{4}\right) \geq 1$, that is, $x_{1}-x_{2}+x_{3}-x_{4} \geq-1$. Then, roughly, a solution will be found (or inconsistency discovered) by deriving new inequations with some variables projected out by performing Chvatal's cut (linear combinations of inequations), in a way indeed quite similar to resolution. A related method is presented in 3, 4, where various heuristics can be used in the choice of the next variable to be eliminated, encoded in an objective function which will guide the search towards an optimal solution. The method will therefore consist in generating a sequence of vectors $X_{1}, \ldots, X_{k}$ such that $X_{k}$ has its elements in $\{0,1\}$ and is an optimal solution (satisfying the initial constraints).

Such a method can perform quite efficiently, especially for big problems, and is more suited to find one solution than all possible solutions.

\subsection{Propagation-based methods.}

These schemes are based on local propagation techniques developed for finite domain constraints. Such techniques stem from Constraint Satisfaction Problems [19, 22, 23, and have been introduced in Constraint Logic Programming by the CHIP language 31. Very close to those methods are the interval arithmetic constraints of BNR-Prolog [5]. The basic idea is to manage a network of constraints between a set of variables which can take values in some finite domains by ensuring local consistency propagation through the constraints linking the variables. Constraint Logic Programming usually only implements, for efficiency reasons, arcconsistency, i.e. propagation of unary constraints (domains of variables), rather than full path-consistency, i.e. propagation of binary constraints, or more general 
full $k$-consistency, i.e. constraints involving $k$ variables. Also a popular technique is to enforce a relaxed, or partial, form of $k$-consistency (also called partial lookahead [16]), which consists in considering and propagating through the constraint network not the full domains of variables but only some approximations of domains, such as the minimal and maximal values. The efficiency of this scheme have been assessed for handling linear equations and inequation in numerical problems, see [31. or [10. Thus some constraints can be consistent with the approximations of current domains of variables but not completely satisfied and should be reconsidered when the domains of the variables they involve are further reduced. Therefore the constraint popagation phase is followed by a so-called labeling phase where variables not yet fixed are incrementally instanciated to some value in their domains (which has usually been reduced in the previous phase). Various heuristics can be incorporated in this labeling phase in order to choose the next variable to instanciate. An instanciation can lead to the (re)activation of some constraints that are not completely satisfied, possibly reducing the domains of other variables. This process continues until some solution is found, i.e. until no suspended constraint needs to be reconsidered anymore. Such techniques are called local consistency because they do not ensure global consistency in general, although for instance arc-consistency is complete for some subsets of binary constraints and $n$-consistency will obviously ensure global consistency of a system of $n$ variables. Note that global consistency means that the problem is solved. Methods based on finite domain propagation techniques are very close in spirit to enumerative methods (especially [21]), but do not use a particular encoding of boolean constraints, and rather reuse a more general module designed for finite domain constraints. Such a boolean solver is integrated in CHIP and performs usually far better than its boolean unification algorithm, being close to specifically developed solvers. clp(B/FD) is another instance of such an approach, as it is based on the clp(FD) solver for finite domain constraints, as is $\operatorname{clp}(\mathrm{B})$, which is used on a specialized low-level constraint based on local propagation.

\subsection{CLP versus dedicated solvers}

It is also worth distinguishing in the above classification between special-purpose boolean solvers, which are intended to take a set of boolean formulas as input, and solvers integrated in CLP languages, which offer much more flexibility by providing a full logic language to state the problem and generate the boolean formulas. PrologIII, CHIP, $\operatorname{clp}(\mathrm{B} / \mathrm{FD})$ and $\mathrm{clp}(\mathrm{B})$ fall into the latter category.

The advantages of being integrated in a full CLP language are as follows. First, the underlying logic language can be used as a metalanguage for stating the boolean constraints, instead of giving an explicit boolean formulations, e.g. a clausal form, which is in general quite complex and rather unreadable. Second, heuristics can be added in the program itself, as opposed to a closed boolean solver with (a finite set of) built-in heuristics. Finally, being fully integrated in a finite domain solver, as in $\operatorname{clp}(\mathrm{B} / \mathrm{FD})$, make it possible for various extensions such as pseudo-booleans [6] or multi-valued logics 33 . Pseudo-boolean constraints are very important because they usually lead to a simpler formulation and because they can provide a much better pruning. 


\section{Formalizing propagation-based solvers}

Let us now detail the formalization of boolean expressions in terms of constraint systems. In this way, we give an operational semantics to the propagation-based boolean solver and prove its equivalence with respect to the declarative semantics of boolean expressions (truth-tables).

\subsection{Constraint Systems}

The simplest way to define constraints is to consider them as first-order formulas interpreted in some non-Herbrand structure [18, in order to take into account the particular semantics of the constraint system. Such declarative semantics is adequate when a non-Herbrand structure exists beforehand and suitably fits the constraint system (e.g. $\mathcal{R}$ for arithmetic constraints), but does not work very well for more practical constraint systems (e.g. finite domains). Obviously, it cannot address any operational issues related to the constraint solver itself. Recently, another formalization has been proposed by [28], which can be seen as a first-order generalization of Scott's information sytems [29]. The emphasis is put on the definition of an entailment relation (noted $\vdash$ ) between constraints, which suffices to define the overall constraint system. Such an approach is of prime importance in the framework of concurrent constraint languages, but is also useful for pure CLP, as it makes it possible to define a constraint system ex nihilo by giving the entailment relation and verifying some basic properties. The entailment relation is given by rules, and we can therefore define a kind of operational semantics of the entailment between constraints. This will be particularly useful when defining our propagation-based boolean constraint system, as the entailment relation will accurately represent how information is propagated between constraints.

Definition [28]

A constraint system is a pair $(D, \vdash)$ satisfying the following conditions:

1. $D$ is a set of first-order formulas closed under conjunction and existential quantification.

2. $\vdash$ is an entailment relation between a finite set of formulas and a single formula satisfying the following inference rules:

$$
\begin{array}{ll}
\Gamma, d \vdash d \text { (Struct) } & \frac{\Gamma_{1} \vdash d \quad \Gamma_{2}, d \vdash e}{\Gamma_{1}, \Gamma_{2} \vdash e} \text { (Cut) } \\
\frac{\Gamma, d, e \vdash f}{\Gamma, d \wedge e \vdash f}(\wedge \vdash) & \frac{\Gamma \vdash d \quad \Gamma \vdash e}{\Gamma \vdash d \wedge e}(\vdash \wedge) \\
\frac{\Gamma, d \vdash e}{\Gamma, \exists X . d \vdash e}(\exists \vdash) & \frac{\Gamma \vdash d[t / X]}{\Gamma \vdash \exists X . d}(\vdash \exists)
\end{array}
$$

In $(\exists \vdash), X$ is assumed not free in $\Gamma, e$.

3. $\vdash$ is generic: that is $\Gamma[t / X] \vdash d[t / X]$ whenever $\Gamma \vdash d$, for any term $t$.

In order to build constraint systems, it suffices to define a pre-constraint system $(D, \vdash)$ satisfying only (Struct), (Cut) and the genericity condition. Existential quantification and conjunction can be added in a straightforward way, as stated by the following theorem. 


\section{Theorem [28]}

Let $\left(D^{\prime}, \vdash^{\prime}\right)$ be a pre-constraint system. Let $D$ be the closure of $D^{\prime}$ under existential quantification and conjunction, and $\vdash$ the closure of $\vdash^{\prime}$ under the basic inference rules. Then $(D, \vdash)$ is a constraint system.

As an important corollary, a constraint system can be constructed even more simply from any first-order theory, i.e. any set of first-order formulas. Consider a theory $T$ and take for $D$ the closure of the subset of formulas in the vocabulary of $T$ under existential quantification and conjunction. Then one defines the entailment relation $\vdash_{T}$ as follows. $\Gamma \vdash_{T} d$ iff $\Gamma$ entails $d$ in the logic, with the extra non-logical axioms of $T$.

Then $\left(D, \vdash_{T}\right)$ can be easily verified to be a constraint system.

Observe that this definition of constraint systems thus naturally encompasses the traditional view of constraints as interpreted formulas.

\subsection{Boolean constraints}

\section{Definition}

Let $\mathcal{V}$ be an enumerable set of variables. A boolean constraint on $\mathcal{V}$ is one of the following formulas:

$$
\operatorname{and}(X, Y, Z), \operatorname{or}(X, Y, Z), \operatorname{not}(X, Y), X=Y, \text { for } X, Y, Z \in \mathcal{V} \cup\{0,1\}
$$

The intuitive meaning of these constraints is: $X \wedge Y \equiv Z, X \vee Y \equiv Z, X \equiv \neg Y$, and $X \equiv Y$. We note $\mathcal{B}$ the set of all such boolean constraints.

Let us now present the rules defining the propagation between boolean constraints.

\section{Definition}

Let $B$ be the first-order theory on $\mathcal{B}$-formulas presented in table 1 .

\begin{tabular}{|ll|}
\hline $0=0$ & $1=1$ \\
$\operatorname{and}(X, Y, Z), X=0 \rightarrow Z=0$ & $\operatorname{and}(X, Y, Z), Y=0 \rightarrow Z=0$ \\
$\operatorname{and}(X, Y, Z), X=1 \rightarrow Z=Y$ & and $(X, Y, Z), Y=1 \rightarrow Z=X$ \\
$\operatorname{and}(X, Y, Z), Z=1 \rightarrow X=1$ & $\operatorname{and}(X, Y, Z), Z=1 \rightarrow Y=1$ \\
& \\
$\operatorname{or}(X, Y, Z), X=1 \rightarrow Z=1$ & $\operatorname{or}(X, Y, Z), Y=1 \rightarrow Z=1$ \\
$\operatorname{or}(X, Y, Z), X=0 \rightarrow Z=Y$ & $\operatorname{or}(X, Y, Z), Y=0 \rightarrow Z=X$ \\
$\operatorname{or}(X, Y, Z), Z=0 \rightarrow X=0$ & $\operatorname{or}(X, Y, Z), Z=0 \rightarrow Y=0$ \\
& \\
$\operatorname{not}(X, Y), X=0 \rightarrow Y=1$ & $\operatorname{not}(X, Y), X=1 \rightarrow Y=0$ \\
$\operatorname{not}(X, Y), Y=0 \rightarrow X=1$ & $\operatorname{not}(X, Y), Y=1 \rightarrow X=0$ \\
\hline
\end{tabular}

Table 1: Boolean propagation theory $B$

Note that it is easy to enrich, if desired, this constraint system by other boolean constraints such as xor (exclusive or), nand (not and), nor (not or), $\Leftrightarrow$ (equivalence), or $\Rightarrow$ (implication) by giving the corresponding rules, but they can also be decomposed into the basic constraints. 
We can now define the entailment relation $\vdash_{B}$ between boolean constraints and the boolean constraint system:

\section{Definitions}

Consider a store $\Gamma$ and a boolean constraint $b$.

$\Gamma \vdash_{B} b$ iff $\Gamma$ entails $b$ with the extra axioms of $B$.

The boolean constraint system is $\left(\mathcal{B}, \vdash_{B}\right)$.

It is worth noticing that the rules of $B$ (and thus $\vdash_{B}$ ) precisely encode the propagation mechanisms that will be used to solve boolean constraints. We have indeed given the operational semantics of the constraint solver in this way.

\subsection{Correctness and completeness of $\left(\mathcal{B}, \vdash_{B}\right)$}

It is important to ensure that our (operationally-defined) constraint system is equivalent to traditional boolean expressions. To do so, we have to prove that our entailment relation derives the same results as the declarative semantics of booleans given by the truth-tables of the and, or and not operators.

\section{Theorem}

The and $(X, Y, Z)$, or $(X, Y, Z)$, and $\operatorname{not}(X, Y)$ constraints are satisfied for some values of $X, Y$ and $Z$ iff the tuple of variables is given by the truth-tables of the corresponding boolean operators.

\section{Proof}

It must be shown that, for and $(X, Y, Z)$ and $\operatorname{or}(X, Y, Z)$, once $X$ and $Y$ are bound to some value, the value of $Z$ is correct, i.e. it is unique (if several rules can be applied, they give the same result) and it is equal to the value given by the corresponding truth-table, and that all rows of the truth-tables are reached.

This can be verified by a straightforward case analysis.

For $\operatorname{not}(X, Y)$ it can be easily shown that for any $X, Y$ is given the opposite value.

\section{Booleans on top of clp(FD)}

The first approach we will present consists of implementing boolean constraints in the constraint logic programming language over finite domains clp(FD), by using the possibility to define new constraints in terms of the unique primitive constraint of the system.

\section{$4.1 \quad c l p(F D)$ in a nutshell}

As introduced in Logic Programming by the CHIP language, clp(FD) [13 is a constraint logic language based on finite domains, where constraint solving is done by propagation and consistency techniques originating from Constraint Satisfaction Problems [19, 23, 34]. The novelty of $c l p(F D)$ is the use of a unique primitive constraint which allows the user to define his own high-level constraints. The black box approach gives way to glass box approach. 


\subsubsection{The constraint $X$ in $r$}

The main idea is to use a single primitive constraint $X$ in $r$, where $X$ is a finite domain (FD) variable and $r$ denotes a range, which can be not only a constant range, e.g. $1 . .10$ but also an indexical range using:

- $\min (Y)$ which represents the minimal value of $Y$ (in the current store),

- $\max (Y)$ which represents the maximal value of $Y$,

- $\operatorname{val}(Y)$ which represents the value of $Y$ as soon as $Y$ is ground.

A fragment of the syntax of this (simple) constraint system is given in table 2 .

\begin{tabular}{|c|c|c|c|}
\hline \multicolumn{4}{|c|}{$\mathrm{c}::=\mathrm{X}$ in $\mathrm{r}$ (constraint) } \\
\hline \multirow[t]{2}{*}{$r$} & $::=$ & $\begin{array}{l}t \ldots t \\
\{t\}\end{array}$ & $\begin{array}{l}\text { (interval range) } \\
\text { (singleton range) }\end{array}$ \\
\hline & & $\ldots$ & \\
\hline \multirow[t]{8}{*}{$t$} & $::=$ & $\mathrm{C}$ & (parameter) \\
\hline & & $\mathrm{n}$ & (integer) \\
\hline & & $\min (\mathrm{X})$ & (indexical $\mathrm{min}$ ) \\
\hline & & $\max (\mathrm{X})$ & (indexical max) \\
\hline & & $\operatorname{val}(\mathrm{X})$ & (delayed value) \\
\hline & & $t+t$ & (addition) \\
\hline & & $t-t$ & (subtraction) \\
\hline & & $t * t$ & (multiplication) \\
\hline
\end{tabular}

Table 2: fragment of the constraint system syntax

The intuitive meaning of such a constraint is: " $X$ must belong to $r$ in any store".

The initial domain of an FD variable is $0 . . \infty$ and is gradually reduced by $X$ in $r$ constraints which replace the current domain of $X\left(D_{X}\right)$ by $D_{X}^{\prime}=D_{X} \cap r$ at each modification of $r$. An inconsistency is detected when $D_{X}^{\prime}$ is empty. Obviously, such a detection is correct if the range denoted by $r$ can only decrease. So, there are some monotone restrictions about the constraints 32 . To deal with the special case of anti-monotone constraints we use the general forward checking propagation mechanism [16] which consists in awaking a constraint only when its arguments are ground (i.e. with singleton domains). In clp(FD) this is achieved using a new indexical term $\operatorname{val}(X)$ which delays the activation of a constraint in which it occurs until $X$ is ground.

As shown in the previous table, it is possible to define a constraint w.r.t. the $\min$ or the $\max$ of some other variables, i.e. reasoning about the bounds of the intervals (partial lookahead 31). clp(FD) also allows operations about the whole domain in order to also propagate the "holes" (full lookahead 31]). Obviously, these possibilities are useless when we deal with boolean variables since the domains are restricted to $0 . .1$. 


\subsubsection{High-level constraints and propagation mechanism}

From $X$ in $r$ constraints, it is possible to define high-level constraints (called user constraints) as Prolog predicates. Each constraint specifies how the constrained variable must be updated when the domains of other variables change. In the following examples $X, Y$ are FD variables and $C$ is a parameter (runtime constant value).

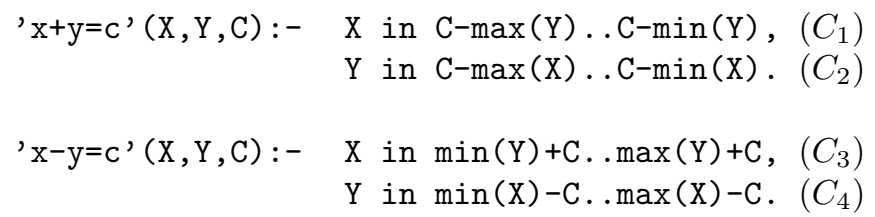

The constraint $x+y=c$ is a classical FD constraint reasoning about intervals. The domain of $X$ is defined w.r.t. the bounds of the domain of $Y$.

In order to show how the propagation mechanism works, let us trace the resolution of the system $\{X+Y=4, X-Y=2\}$ (translated via ' $\mathrm{x}+\mathrm{y}=\mathrm{c}^{\prime}(\mathrm{X}, \mathrm{Y}, 4)$ and ' $\mathrm{X}-\mathrm{y}=\mathrm{c}$ ' $(\mathrm{X}, \mathrm{Y}, 2))$ :

After executing ' $\mathrm{x}+\mathrm{y}=\mathrm{c}$ ' $(\mathrm{X}, \mathrm{Y}, 4)$, the domain of $X$ and $Y$ are reduced to $0 . .4\left(C_{1}\right.$ is in the current store: $X$ in $-\infty . .4, C_{2}: Y$ in $\left.-\infty . .4\right)$. And, after executing ${ }^{\prime} \mathrm{x}-\mathrm{y}=\mathrm{c}$ ' $(\mathrm{X}, \mathrm{Y}, 2)$, the domain of $X$ is reduced to $2 . .4\left(C_{3}: X\right.$ in $\left.2 . .6\right)$, which then reduces the domain of $Y$ to $0 . .2\left(C_{4}: Y\right.$ in $\left.0 . .2\right)$.

Note that the unique solution $\{X=3, Y=1\}$ has not yet been found. Indeed, in order to efficiently achieve consistency, the traditional method (arc-consistency) only checks that, for any constraint $C$ involving $X$ and $Y$, for each value in the domain of $X$ there exists a value in the domain of $Y$ satisfying $C$ and vice-versa. So, once arc-consistency has been achieved and the domains have been reduced, an enumeration (called labeling) has to be done on the domains of the variables to yield the exact solutions. Namely, $X$ is assigned to one value in $D_{X}$, its consequences are propagated to other variables, and so on. If an inconsistency arises, other values for $X$ are tried by backtracking. Note that the order used to enumerate the variables and to generate the values for a variable can improve the efficiency in a very significant manner (see heuristics in 31]).

In our example, when the value 2 is tried for $X, C_{2}$ and $C_{4}$ are awoken (because they depend on $X) . C_{2}$ sets $Y$ to 2 and $C_{4}$ detects the inconsistency when it tries to set $Y$ to 0 . The backtracking reconsiders $X$ and tries value 3 and, as previously, $C_{2}$ and $C_{4}$ are reexecuted to set (and check) $Y$ to 1 . The solution $\{X=3, Y=1\}$ is then obtained.

\subsubsection{Optimizations}

The uniform treatment of a single primitive for all complex user constraints lead to a better understanding of the overall constraint solving process and allows for (a few) global optimizations, as opposed to the many local and particular optimizations hidden inside the black-box. When a constraint $X$ in $r$ has been reexecuted, if $D_{X}^{\prime}=$ $D_{X}$ it was useless to reexecute it (i.e. it has neither failed nor reduced the domain of $X$ ). Hence, we have designed three simple but powerful optimizations for the $X$ in $r$ constraint [13, 10] which encompass many previous particular optimizations for FD constraints: 
- some constraints are equivalent so only the execution of one of them is needed. In the previous example, when $C_{2}$ is called in the store $\{X$ in $0 . .4, Y$ in $0 . . \infty\}$ $Y$ is set to 0..4. Since the domain of $Y$ has been updated, all constraints depending on $Y$ are reexecuted and $C_{1}(X$ in $0 . .4)$ is awoken unnecessarily $\left(C_{1}\right.$ and $C_{2}$ are equivalent).

- it is useless to reexecute a constraint as soon as it is entailed. In clp(FD), only one approximation is used to detect the entailment of a constraint $X$ in $r$ which is " $X$ is ground". So, it is useless to reexecute a constraint $X$ in $r$ as soon as $X$ is ground.

- when a constraint is awoken more than once from several distinct variables, only one reexecution is necessary. This optimization is obvious since the order of constraints, during the execution, is irrelevant for correctness.

These optimizations make it possible to avoid on average $50 \%$ of the total number of constraint executions on a traditional set of FD benchmarks (see 13, 10, for full details) and up to $57 \%$ on the set of boolean benchmarks presented below.

\subsubsection{Performances}

Full implementation results about the performances of clp(FD) can be found in 13, 10, and show that this "glass-box" approach is sound and can be competitive in terms of efficiency with the more traditional "black-box" approach of languages such as CHIP. On a traditional set of benchmark programs, mostly taken from 31, the clp(FD) engine is on average about four times faster than the CHIP system, with peak speedup reaching eight.

\subsection{Building clp(B/FD)}

In this section we specify the constraint solver, i.e. we define a user constraint for each boolean constraint presented above. We then prove the correctness and completeness of this solver, and show how it really encodes the "operational semantics" defined by theory $B$.

\subsubsection{Designing the constraints}

The design of the solver only consists in defining a user constraint for each boolean constraint. As the constraint $X$ in $r$ makes it possible to use arithmetic operations on the bounds of a domain, we use some mathematical relations satisfied by the boolean constraints: 


$$
\begin{array}{lll}
\operatorname{and}(X, Y, Z) \quad \text { satisfies } & Z=X \times Y \\
& & Z \leq X \leq Z \times Y+1-Y \\
& & Z \leq Y \leq Z \times X+1-X \\
& & \\
\operatorname{or}(X, Y, Z) \quad \text { satisfies } & Z=X+Y-X \times Y \\
& & Z \times(1-Y) \leq X \leq Z \\
& Z \times(1-X) \leq Y \leq Z \\
\operatorname{not}(X, Y) \quad \text { satisfies } & X=1-Y \\
& & Y=1-X
\end{array}
$$

The definition of the solver is then quite obvious and presented in table 3 It only encodes the above relations.

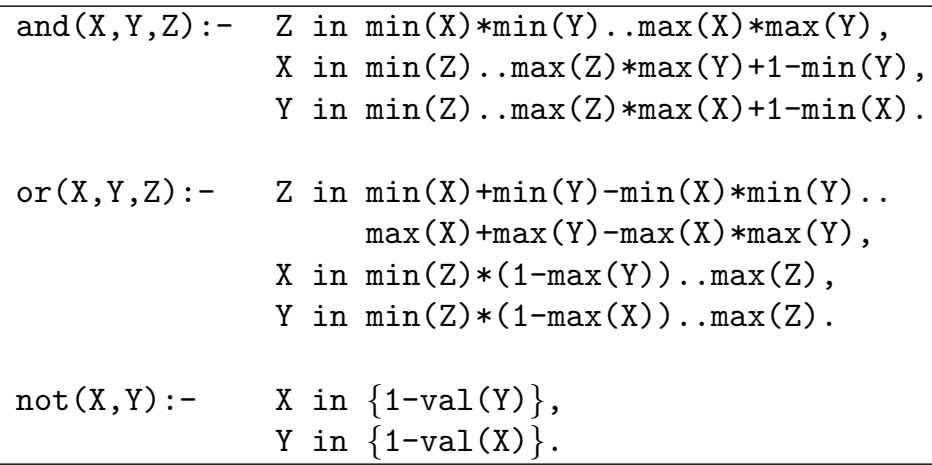

Table 3: The boolean solver definition

\subsubsection{Correctness and completeness of $c l p(B / F D)$}

\section{Theorem}

The and, or, and not user constraints are correct and complete.

\section{Proof}

The proof of correctness consists in showing that each $\{0,1\}$ tuple satisfying the relations defined above is an element of the appropriate truth-table. Completeness w.r.t declarative semantics can be easily proved conversely, but we are mainly interested in showing that each time a rule of $B$ can fire, the store is reduced as specified by the rule. Namely, any tuple of variables satisfies the corresponding mathematical relations enforced by the constraint solver. Here again, a case analysis proves the result. For instance if $\operatorname{and}(X, Y, Z), Y=1 \rightarrow Z=X$ fires, $Z \leq X \leq Z \times Y+1-Y$ is verified in the resulting store.

\section{Performance evaluations of $\operatorname{clp}(\mathrm{B} / \mathrm{FD})$}

\subsection{The benchmarks}

In order to test the performances of $c l p(B / F D)$ we have tried a set of traditional boolean benchmarks: 
- schur: Schur's lemma. The problem consists in finding a 3-coloring of the integers $\{1 \ldots n\}$ such that there is no monochrome triplet $(x, y, z)$ where $x+y=z$. The formulation uses $3 \times n$ variables to indicate, for each integer, its color. This problem has a solution iff $n \leq 13$.

- pigeon: the pigeon-hole problem consists in putting $n$ pigeons in $m$ pigeonholes (at most 1 pigeon per hole). The boolean formulation uses $n \times m$ variables to indicate, for each pigeon, its hole number. Obviously, there is a solution iff $n \leq m$.

- queens: place $n$ queens on a $n \times n$ chessboard such that there are no queens threatening each other. The boolean formulation uses $n \times n$ variables to indicate, for each square, if there is a queen on it.

- ramsey: find a 3-coloring of a complete graph with $\mathrm{n}$ vertices such that there is no monochrome triangles. The formulation uses 3 variables per edge to indicate its color. There is a solution iff $n \leq 16$.

All solutions are computed unless otherwise stated. The results presented below for clp(B/FD) do not include any heuristics and have been measured on a Sun Sparc 2 (28.5 Mips). The following section compares clp(B/FD) with the commercial version of CHIP. We have chosen CHIP for the main comparison because it is a commercial product and a CLP language (and not only a constraint solver) and thus accepts the same programs as $\operatorname{clp}(\mathrm{B} / \mathrm{FD})$. Moreover, it also uses a boolean constraint solver based on finite domain ${ }^{1}$. We also compare clp(B/FD) with other specific constraint solvers.

\section{$5.2 \operatorname{clp}(B / F D)$ versus CHIP}

Times for CHIP were also measured on a Sun Sparc 2. Exactly the same programs were run on both systems.

The average speedup of $c l p(B / F D)$ w.r.t. CHIP is around a factor of eight, with peak speedup reaching two orders of magnitude, see table 4. This factor of eight can be compared with the factor of four that we have on the traditional FD benchmarks. The main reasons for this gap could be that in CHIP, booleans are written on an existing solver whereas we have developed an appropriate solver thanks to the $X$ in $r$ primitive, and that we have global optimizations for primitive constraints from which all user constraints can benefit.

\section{$5.3 \mathrm{clp}(\mathrm{B} / \mathrm{FD})$ versus the rest}

In this section, we compare $c l p(B / F D)$ with other specific boolean solvers. These solvers are not programming languages, they accept a set of constraints as input and solve it. So there are as many formulations as problem instances. On the other hand, $\operatorname{clp}(\mathrm{B} / \mathrm{FD})$ generates constraints at runtime (the overhead thus introduced is limited to $20 \%$, so we do not need to worry too much about that). Another important point to mention is that were not able to run exactly the same programs, and we have used time measurements provided by the referenced papers (which usually incorporate a large number of heuristics).

\footnotetext{
${ }^{1}$ the other solver of CHIP, based on boolean unification, became quickly unpracticable: none of the benchmarks presented here could even run with it, due to memory limitations.
} 


\begin{tabular}{||l||r|r|r||}
\hline Program & \multicolumn{1}{|c|}{$\begin{array}{c}\text { CHIP } \\
\text { Time (s) }\end{array}$} & $\begin{array}{c}\text { clp (B/FD) } \\
\text { Time (s) }\end{array}$ & \multicolumn{1}{c|}{ CHIP } \\
\hline \hline schur 13 1 FD)
\end{tabular}

Table 4: clp(B/FD) versus CHIP 


\subsection{1 $c l p(B / F D)$ versus BDD methods}

Adia is an efficient boolean constraint solver based on the use of BDDs. Time measurements presented below are taken from [27, in which four different heuristics are tried on a Sun Sparc IPX (28.5 Mips). We have chosen the worst and the best of these four timings for Adia. Note that the BDD approach computes all solutions and is thus unpracticable when we are only interested in one solution for big problems such as queens for $n \geq 9$ and schur for $n=30$. Here again, clp(B/FD) has very good speedups (see table 5 , where the sign $\downarrow$ before a number means in fact a slowdown of $\operatorname{clp}(\mathrm{B} / \mathrm{FD})$ by that factor).

\begin{tabular}{|c|c|c|c|c|c|}
\hline Program & $\begin{array}{l}\text { Bdd worst } \\
\text { Time (s) }\end{array}$ & $\begin{array}{l}\text { Bdd best } \\
\text { Time (s) }\end{array}$ & $\begin{array}{c}\text { clp (B/FD) } \\
\text { Time (s) }\end{array}$ & $\frac{\text { Bdd worst }}{\operatorname{clp}(B / F D)}$ & $\frac{\text { Bdd best }}{\text { clp }(B / F D)}$ \\
\hline "schur 13 & 3.260 & $\overline{1.110}$ & $\overline{0.100}$ & "32.60 & $\overline{11.10}$ \\
\hline schur 14 & 5.050 & 1.430 & 0.100 & 50.50 & 14.30 \\
\hline pigeon $7 / 6$ & 1.210 & 0.110 & 0.310 & 3.90 & $\downarrow 2.81$ \\
\hline pigeon $7 / 7$ & 3.030 & 0.250 & 2.660 & 1.13 & $\downarrow 10.64$ \\
\hline pigeon $8 / 7$ & 4.550 & 0.310 & 2.220 & 2.04 & $\downarrow 7.16$ \\
\hline pigeon $8 / 8$ & 15.500 & 0.580 & 24.240 & $\downarrow 1.56$ & $\downarrow 41.79$ \\
\hline queens 6 & 2.410 & 1.010 & 0.060 & 40.16 & 16.83 \\
\hline queens 7 & 12.030 & 4.550 & 0.170 & 70.76 & 26.76 \\
\hline queens 8 & 59.210 & 53.750 & 0.490 & 120.83 & 109.69 \\
\hline
\end{tabular}

Table 5: $\operatorname{clp}(\mathrm{B} / \mathrm{FD})$ versus a BDD method

\subsection{2 $c l p(B / F D)$ versus enumerative methods}

[26] provides time measurements for an enumerative method for boolean unification on a Sun 3/80 (1.5 Mips). We normalized these measurements by a factor of 1/19. The average speedup is 6.5 (see table 6).

\begin{tabular}{||l||r|r|r||}
\hline Program & $\begin{array}{c}\text { Enum } \\
\text { Time (s) }\end{array}$ & $\begin{array}{c}\text { clp (B/FD) } \\
\text { Time (s) }\end{array}$ & \multicolumn{1}{c||}{ Enum } \\
\hline \hline schur 13 13 FD) \\
schur 14 & 0.810 & 0.100 & 8.10 \\
\hline pigeon 5/5 & 0.880 & 0.100 & 8.80 \\
pigeon 6/5 & 0.210 & 0.060 & 3.50 \\
pigeon 6/6 & 2.290 & 0.050 & 2.40 \\
pigeon 7/6 & 0.840 & 0.360 & 6.36 \\
\hline queens 7 & 0.370 & 0.310 & 2.70 \\
queens 8 & 1.440 & 0.540 & 2.17 \\
queens 9 & 6.900 & 2.140 & 2.66 \\
\hline
\end{tabular}

Table 6: $c l p(B / F D)$ versus an enumerative method 


\subsection{3 $c l p(B / F D)$ versus a boolean local consistency method}

Here, we refer to 21$]$ in which are presented the results of a boolean constraint solver based on the use of local consistency techniques. Times are given on a Macintosh $\mathrm{SE} / 30$ equivalent to a Sun 3/50 (1.5 Mips). We normalized them with a factor of $1 / 19$. This solver includes two labeling heuristics, the most important being the ability to dynamically order the variables w.r.t. the number of constraints still active on them. On the other hand, $\operatorname{clp}(\mathrm{B} / \mathrm{FD}$ ) only uses a static order (standard labeling).

An interesting point is that the factors are quite constant within a class of problem. $\mathrm{clp}(\mathrm{B} / \mathrm{FD})$ is slower on the schur benchmark by a factor of 1.4 , three times faster on pigeon and four times faster on queens (see table 7 for more details). We conjecture that this is because both solvers certainly perform much the same pruning, although they are based on very different data-structures for the constraints and constraint network.

\begin{tabular}{|c|c|c|c|}
\hline Program & $\begin{array}{c}\text { BCons } \\
\text { Time (s) }\end{array}$ & $\begin{array}{l}\text { clp (B/FD) } \\
\text { Time (s) }\end{array}$ & $\frac{\text { BCons }}{\operatorname{clp}(\mathrm{B} / \mathrm{FD})}$ \\
\hline schur 13 & 0.070 & 0.100 & $\bar{\downarrow} 1.42$ \\
\hline schur 14 & 0.080 & 0.100 & $\downarrow 1.25$ \\
\hline pigeon $7 / 6$ & 0.870 & 0.310 & 2.80 \\
\hline pigeon $7 / 7$ & 7.230 & 2.660 & 2.71 \\
\hline pigeon $8 / 7$ & 6.820 & 2.220 & 3.07 \\
\hline pigeon $8 / 8$ & 67.550 & 24.240 & 2.78 \\
\hline queens 8 & 1.810 & 0.540 & 3.35 \\
\hline queens 9 & 7.752 & 2.140 & 3.62 \\
\hline queens 10 & 32.720 & 8.270 & 3.95 \\
\hline queens 14 1st & 3.140 & 0.870 & 3.60 \\
\hline queens $161 \mathrm{st}$ & 17.960 & 3.280 & 5.47 \\
\hline
\end{tabular}

Table 7: $\operatorname{clp}(\mathrm{B} / \mathrm{FD})$ versus a boolean local consistency method

\subsection{4 $\quad \mathrm{clp}(\mathrm{B} / \mathrm{FD})$ versus an Operational Research method}

We will compare here with the FAST93 method [4, which is based on 0-1 progamming techniques from Operational Research. The time measurements are given for a Sparc Station 1+ (18 MIPS), and therefore normalized by a factor 1/1.6 (see table 8). It should be noted that on the benchmark problems, only the first solution is computed. For the pigeon problem, FAST93 has good performances when the problem is unsatisfiable (even for large values), i.e. when there are more pigeons than holes $(N>M)$. This is because the method can derive quickly that this inequality is not satisfied. The pure boolean formulation that we have tested with clp(B/FD) will not have as good results for larger values, but it is very easy to add a non-boolean constraint $N<M$ (that will detect inconsistency immediately), because the system is embedded in a full finite domain solver. Observe that this would not be possible in a pure boolean solver, and this explains why we do not give this result in our comparisons. 


\begin{tabular}{|c|c|c|c|}
\hline & FAST93 & $\operatorname{clp}(\mathrm{B} / \mathrm{FD})$ & FAST93 \\
\hline Program & Time $(\mathrm{s})$ & Time (s) & $\overline{\operatorname{clp}(B / F D)}$ \\
\hline pigeon $7 / 7$ 1st & 0.250 & 0.020 & 12.50 \\
\hline pigeon $8 / 7$ 1st & 1.940 & 2.220 & $\downarrow 1.14$ \\
\hline pigeon $8 / 8$ 1st & 0.630 & 0.030 & 21 \\
\hline pigeon $9 / 8$ 1st & 4.230 & 20.190 & $\downarrow 4.77$ \\
\hline pigeon $9 / 9$ 1st & 0.690 & 0.040 & 17.25 \\
\hline ramsey 10 1st & 11.500 & 0.110 & 104.54 \\
\hline ramsey 12 1st & 81.440 & 0.190 & 428.42 \\
\hline
\end{tabular}

Table 8: clp(B/FD) versus an Operational Research method

\section{A dedicated boolean solver : $c \operatorname{lp}(B)$}

In the above section we have seen that local propagation techniques are a good way of dealing efficiently with boolean problems and particularly clp(FD) thanks to its low-level $X$ in $r$ primitive. However, only a very restricted subset of the possibilities offered by $X$ in $r$ was used when defining clp(B/FD). Here we are interested in designing a specific propagation-based boolean solver, according to the glass-box paradigm, which will be called $\operatorname{clp}(B)$. This work is interesting for many reasons. First, it will allow us to evaluate the overhead of clp(FD) when only a restricted part of $X$ in $r$ is used. Second, it will help to argue more precisely why local propagation techniques are a very efficient way to deal with boolean constraints in general. Third, it will present a surprisingly simple instruction set which will make it possible to integrate boolean constraints in any Prolog compiler. It is worth noticing that all well-known boolean solvers (CHIP, PrologIII, etc) are based on the black-box approach, i.e. nobody knows exactly what there is inside these solvers, except [12] which presents a glass-box (re)construction of a boolean solver based on BDDs. From a design point of view, $\operatorname{clp}(B)$ is very similar to $c l p(F D)$. It is based on a low-level primitive constraint $l_{0}<=l_{1}, \ldots, l_{n}$ and it offers the possibility to define user constraints as Prolog predicates. Complex boolean constraints are also translated at compile-time by a preprocessor.

\subsection{The primitive constraint $l_{0}<=l_{1}, \ldots, l_{n}$}

Since the initial domain of a boolean variable is $0 . .1$ it can be reduced only once. A constraint is only triggered when some of its variables have become ground, and, if this activation is useful, then the constrained variable will also become ground. Thus, the more appropriate primitive must allow us to express propagation rules which look like "as soon as $X$ is false then set $Z$ to false" and "as soon as both $X$ and $Y$ are true then set $Z$ to true" (for and $(\mathrm{X}, \mathrm{Y}, \mathrm{Z})$ ). Note the difference with the clp(B/FD) formulation where the primitive $X$ in $r$ was used in a computational way to calculate the value ( 0 or 1$)$ to assign. The behavior of this primitive is very similar to the ask definition of and $(\mathrm{X}, \mathrm{Y}, \mathrm{Z})$ presented in 33. Thus, we propose a primitive constraint $l_{0}<=l_{1}, \ldots, l_{n}$ where each $l_{i}$ is either a positive literal $(X)$ or a negative literal $(-X)$ (see table 9 for a description of the syntax).

Associated to each literal $l_{i}$ we define $X_{i}$ as its variable and Bvalue $e_{i}$ as its truthvalue. More precisely if $l_{i} \equiv-X$ or $l_{i} \equiv X$ then $X_{i}=X$. Similarly if $l_{i} \equiv-X$ $\left(\right.$ resp. $\left.l_{i} \equiv X\right)$ then Bvalue $_{i}=0$ (resp. Bvalue $\left.{ }_{i}=1\right)$. 


\begin{tabular}{|ccc|}
\hline $\mathrm{C}::=$ & $1<=[1, \ldots, 1]$ & (constraint) \\
$1::=$ & $\mathrm{X}$ & \\
& $-\mathrm{X}$ & $\begin{array}{l}\text { (positive literal) } \\
\text { (negative literal) }\end{array}$ \\
\hline
\end{tabular}

Table 9: Syntax of the constraint $l_{0}<=l_{1}, \ldots, l_{n}$

The intuitive meaning of $l_{0}<=l_{1}, \ldots, l_{n}$ being " $l_{0}$ must be entailed in any store which entails $l_{1} \wedge \ldots \wedge l_{n}$ " where $l_{i}$ is entailed in a store iff $X_{i}=$ Bvalue $_{i}$ is entailed in this store.

Without any loss of generality, we can consider there is only either one or two literals in the body of the primitive constraint. Indeed, the case $n=0$ comes down to unify $X_{0}$ to Bvalue $_{0}$ (see section 6.3.1) and the case $n>2$ can be rewritten by replacing $l_{0}<=\left[l_{1}, l_{2}, l_{3}, \ldots, l_{n}\right]$ by $l_{0}<=\left[l_{1}, I_{2}\right], I_{2}<=\left[l_{2}, I_{3}\right], \ldots, I_{n-1}<=\left[l_{n-1}, l_{n}\right]$, where each $I_{k}$ is a distinct new boolean variable. In $\operatorname{clp}(\mathrm{B})$ a preprocessor is used for these code-rewriting. This decomposition will allow us to implement very efficiently the tell operation as shown below since only the two cases $n=1$ and $n=2$ remain.

\subsection{Defining the constraints}

As previously done in order to build $\operatorname{clp}(\mathrm{B} / \mathrm{FD})$ we here define a user constraint for each boolean constraint. It is worth noticing that the $l_{0}<=l_{1}, \ldots, l_{n}$ primitive allows us to directly encode the propagation rules presented above ${ }^{2}$ The definition of the solver is then quite obvious and presented in table 10 .

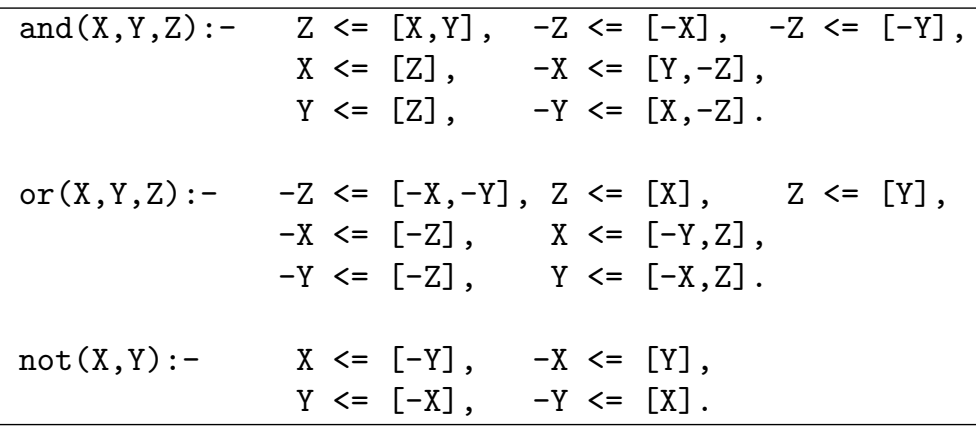

Table 10: The boolean solver definition

\subsection{Integration of $l_{0}<=l_{1}, \ldots, l_{n}$ into the WAM}

Let us now specify the abstract instruction set needed to implement the boolean constraint solver, i.e. the unique constraint $l_{0}<=l_{1}, \ldots, l_{n}$, into the standard abstract machine used in Logic Programming, namely the Warren Abstract Machine. See [35, 1] for a comprehensive introdution to the WAM.

\footnotetext{
${ }^{2}$ the proofs of correctness and completeness are obvious and left to the reader...
} 


\subsubsection{Modifying the WAM for boolean variables}

Here, we explain the necessary modifications of the WAM to manage a new data type: boolean variables. They will be located in the heap, and an appropriate tag is introduced to distinguish them from Prolog variables. Dealing with boolean variables slightly affects data manipulation, unification, indexing and trailing instructions.

Data manipulation. Boolean variables, as standard WAM unbound variables, cannot be duplicated (unlike it is done for terms by structure-copy). For example, loading an unbound variable into a register consists of creating a binding to the variable whereas loading a constant consists of really copying it. In the standard WAM, thanks to self-reference representation for unbound variables, the same copy instruction can be used for both of these kinds of loading. Obviously, a boolean variable cannot be represented by a self-reference, so we must take care of this problem. When a source word $\mathrm{W}_{s}$ must be loaded into a destination word $\mathrm{W}_{d}$, if $\mathrm{W}_{s}$ is a boolean variable then $\mathrm{W}_{d}$ is bound to $\mathrm{W}_{s}$ or else $\mathrm{W}_{s}$ is physically copied into $\mathrm{W}_{d}$.

Unification. A boolean variable $X$ can be unified with:

- an unbound variable $Y: Y$ is just bound to $X$,

- an integer $n$ : if $n=0$ or $n=1$ the pair $(X, n)$ is enqueued and the consistency procedure is called (see sections 6.3.3 and 6.3.4.

- another boolean variable $Y$ : equivalent to $X<=[Y],-X<=[-Y]$, $Y<=[X]$ and $-Y<=[-X]^{3}$.

Indexing. The simplest way to manage a boolean variable is to consider it as an ordinary unbound variable and thus try all clauses.

Trailing In the WAM, unbound variables only need one word (whose value is fully defined by their address thanks to self-references), and can only be bound once, thus trailed at most once. When a boolean variable is reduced (to an integer $n=0 / 1$ ) the tagged word $\left\langle\mathrm{BLV},{ }_{-}\right\rangle$(see section 6.3.2) is replaced by $<$INT, $\left.\mathrm{n}\right\rangle$ and the tagged word $\left\langle\mathrm{BLV},{ }_{-}>\right.$may have to be trailed. So a value-trail is necessary. Hence we have two types of objects in the trail: one-word entry for standard Prolog variables, two-word entry for trailing one previous value.

\subsubsection{Data structures for constraints}

clp(B) uses an explicit queue to achieve the propagation (i.e. each triggered constraint is enqueued). It is also possible to use an implicit propagation queue as discussed in [10]. The register BP (Base Pointer) points to the next constraint to execute, the register TP (Top Pointer) points to the next free cell in the queue. The other data structure concerns the boolean variable. The frame of a boolean variable $X$ is shown in table 11 and consists of:

- the tagged word,

\footnotetext{
${ }^{3}$ we will describe later how constraints are managed.
} 
- the list of constraints depending on $X$. For reasons of efficiency two lists are used: constraints depending on $-X$ (Chain_o) and constraints depending on $X$ (Chain_1).

\begin{tabular}{|c|c|c|}
\hline \multicolumn{2}{|c|}{ Chain_1 } & \multirow{3}{*}{$\begin{array}{l}\text { (pointer to a } R_{-} \text {Frame) } \\
\text { (pointer to a R_Frame) }\end{array}$} \\
\hline & ain_0 & \\
\hline BLV & unused & \\
\hline
\end{tabular}

Table 11: Boolean variable frame (B_Frame)

Since there are at most 2 literals in the body of a constraint $c \equiv l_{0}<=l_{1}, \ldots, l_{n}$, if $c$ depends on $X$ (i.e $X_{1}=X$ or $X_{2}=X$ ) it is possible to distinguish the case $n=1$ from the case $n=2$. Intuitively, in the case $n=1$ the constraint $c$ can be solved as soon as $X$ is ground while $c$ can still suspend until the other variable is ground in the case $n=2$ (see section 6.3 .4 for more details). So, the case $n=2$ requires more information about the constraint to trigger since it is necessary to check the other variable before executing it. The frame associated to a record (R_Frame) of the list Chain_0/1 consists of:

- the address of the boolean which is constrained (i.e. $X_{0}$ ),

- the value to affect (i.e. Bvalue $)$,

- only if $n=2$ : the address of the other involved boolean variable

- only if $n=2$ : the value to be satisfied by the other involved variable

Table 12 summarizes the contents of a R_Frame.

It is worth noting that, in the case $n=2$, a record is necessary in the appropriate list of $X_{1}$ with a pointer to $X_{2}$ and also in the appropriate list of $X_{2}$ with a pointer to $X_{1}$. This "duplication" is very limited since it involves only 2 additional words. This is enhanced in figure 2 which shows the data structures involved in the constraint $\mathrm{Z}<=[-\mathrm{X}, \mathrm{Y}]$ (which could be used in the definition of $\mathrm{xor}(\mathrm{X}, \mathrm{Y}, \mathrm{Z})$ ). The alternate solution would use 1 additional word to count the number of variables which suspend (the constraint being told as soon as this counter equals 0 ).

\begin{tabular}{|c|c|}
\hline Bvalue_2 & $\begin{array}{l}\text { (only used } \\
\text { / if Bloc2_Flag is true) } \\
\text { (case } n=2 \text { ?) }\end{array}$ \\
\cline { 1 - 1 } Blv_2_Adr & \\
\cline { 1 - 1 } Bloc2_Flag & $\begin{array}{l}\text { (a pointer to a B_Frame) } \\
\text { Tell_Bvalue }\end{array}$ \\
\cline { 1 - 1 } Tell_Blv_Adr & (a pointer to a R_Frame) \\
\hline Next_Record & \\
\hline
\end{tabular}

Table 12: Record Frame (R_Frame) 


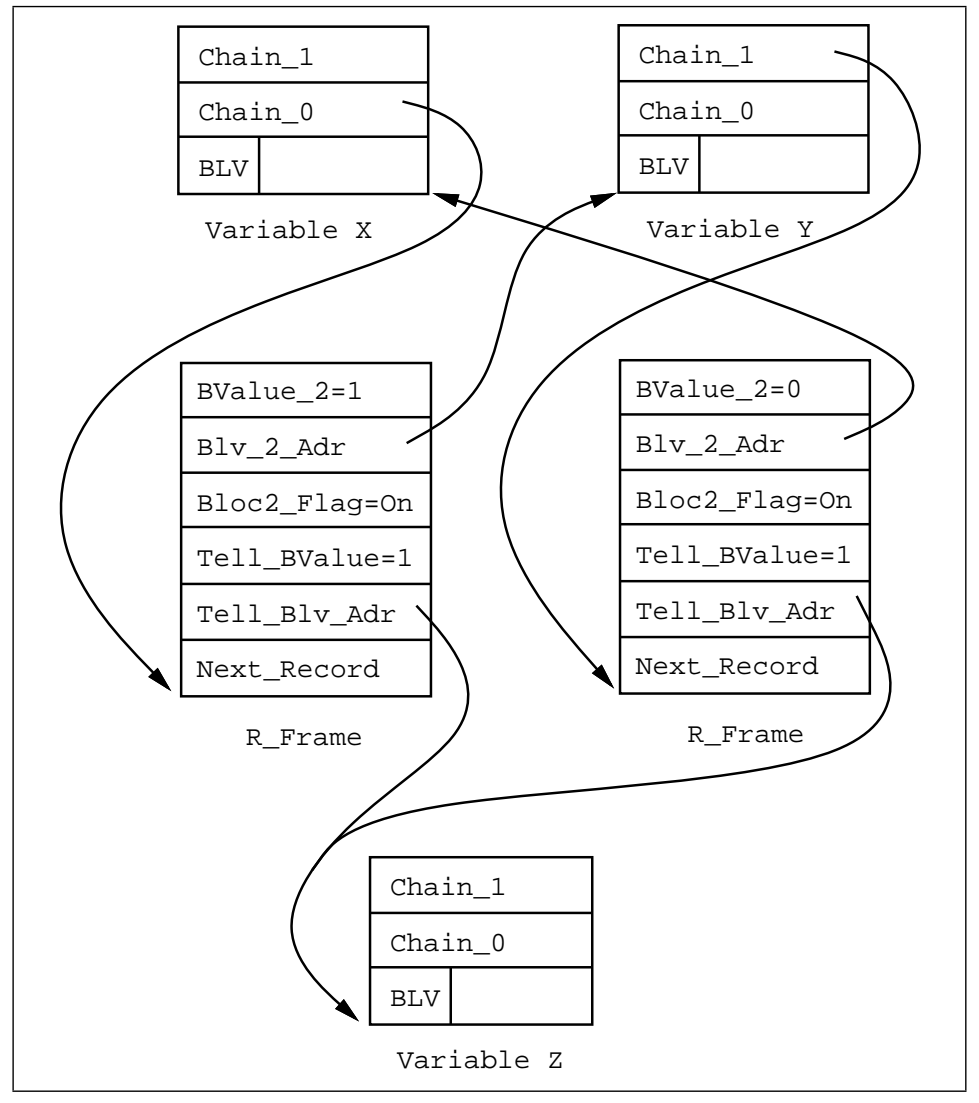

Figure 2: Data structures involved in the constraint $\mathrm{Z}<=[-\mathrm{X}, \mathrm{Y}]$ 


\subsubsection{Compilation scheme and instruction set}

The compilation of a constraint $l_{0}<=l_{1}, \ldots, l_{n}$ consists of two parts:

- loading $X_{0}, \ldots, X_{n}$ into WAM temporaries (i.e. Xi registers),

- installing and telling the constraint, i.e. creating the necessary R_Frame(s), detecting if the body of the constraint is currently entailed by the store (see section 6.1) to enqueue the pair $\left(X_{0}\right.$, Bvalue $\left._{0}\right)$ and to call the consistency procedure (described in section 6.3.4).

Loading instructions are:

b_load_variable $(\mathrm{Vi}, \mathrm{Xj})$

binds Vi to a boolean variable created on top of the heap and puts its address into $\mathrm{Xj}$.

b_load_value $(\mathrm{Vi}, \mathrm{Xj})$

let $\mathrm{w}$ be the dereferenced word of $\mathrm{Vi}$, if it is:

- an unbound variable: similar to b_load_variable (w, $\mathrm{Xj}$ ).

- an integer $n$ : fails if $n \neq 0$ and $n \neq 1$ or else $n$ is pushed on the heap and its address is stored into $\mathrm{Xj}$.

- a boolean variable: its address is stored into $\mathrm{Xj}$.

Install and telling instructions (defined in the case $n=1$ or $n=2$ ) are:

b_install_and_tell_cstr1 (X0, bvalue0, X1, bvalue1)

two cases depending on $\mathrm{X} 1$ :

- $\mathrm{X} 1$ is an integer: if $\mathrm{X} 1=\mathrm{bvalue} 1,(\mathrm{X0}, \mathrm{bvalue})$ is enqueued and the consistency procedure called (else the constraint succeeds immediately as the premise is false).

- X1 is a boolean variable: an R_Frame (created on the top of the heap) is added to the appropriate list of $\mathrm{X} 1$ recording $\mathrm{X} 0$ and bvalue 0 .

b_install_and_tell_cstr2 (X0, bvalue $0, \mathrm{X} 1$, bvalue $1, \mathrm{X} 2$, bvalue 2 )

three cases depending on $\mathrm{X} 1$ and $\mathrm{X} 2$ :

- $\mathrm{X} 1$ is an integer:

it behaves like b_install_and_tell_cstr1 (X0, bvalue0, X2, bvalue2).

- $\mathrm{X} 2$ is an integer:

it behaves like b_install_and_tell_cstr1 (X0, bvalue0, X1, bvalue1).

- X1 and X2 are two boolean variables: an R_Frame (created on the top of the heap) is added to the appropriate list of $\mathrm{X} 1$ recording $\mathrm{X} 0$, bvalue 0 and $\mathrm{X} 2$, bvalue2, and similarly an R_Frame is added to the appropriate list of $\mathrm{X} 2$ recording $\mathrm{X} 0$, bvalue 0 and $\mathrm{X} 1$, bvalue1.

It is worth noticing that only 4 instructions are needed to implement this boolean solver into the WAM. The extension is really minimal. Our experience has shown that in this way only a few days are necessary to incorporate boolean constraints into a Prolog compiler whose sources are well-known.

Table 13 shows an example of code generated for the user constraint and $(\mathrm{X}, \mathrm{Y}, \mathrm{Z})$. 


\begin{tabular}{|c|c|c|}
\hline and/3: & 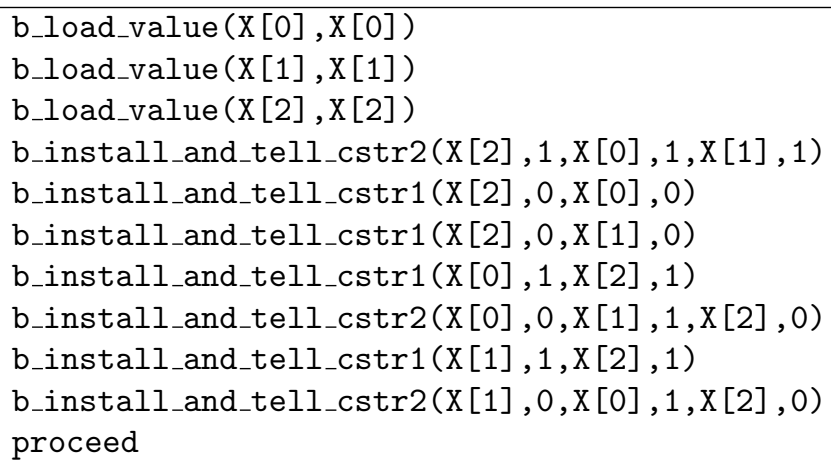 & $\begin{aligned} & \mathrm{X}(0)=\operatorname{address} \text { of } \mathrm{X} \\
& \mathrm{X}(1)=\operatorname{address} \text { of } \mathrm{Y} \\
& \mathrm{X}(2)=\operatorname{address} \text { of } \mathrm{Z} \\
& \mathrm{Z}<=[\mathrm{X}, \mathrm{Y}] \\
&-\mathrm{Z}<=[-\mathrm{X}] \\
&-\mathrm{Z}<=[-\mathrm{Y}] \\
& \mathrm{X}<=[\mathrm{Z}] \\
&-\mathrm{X}<=[\mathrm{Y},-\mathrm{Z}] \\
& \mathrm{Y}<=[\mathrm{Z}] \\
&-\mathrm{Y}<=[\mathrm{X},-\mathrm{Z}] \\
& \text { Prolog return }\end{aligned}$ \\
\hline
\end{tabular}

Table 13: Code generated for and (X, Y, Z)

\subsubsection{The consistency procedure}

This procedure is responsible for ensuring the consistency of the store. It repeats the following steps until the propagation queue is empty (or until a failure occurs): Let $(X, B v a l u e)$ be the pair currently pointed by BP.

- If $X$ is an integer, there are two possibilities:

- $X=$ Bvalue: success (Check $O k$ )

$-X \neq$ Bvalue: failure (Fail)

- else the boolean variable $X$ is set to Bvalue (Reduce) and each constraint depending on $X$ (i.e. each record of Chain_Bvalue) is reconsidered as follows:

- case $n=1$ : the pair $\left(X_{0}\right.$, Bvalue $\left._{0}\right)$ is enqueued.

- case $n=2$ : let us suppose that $X=X_{1}$, the case $X=X_{2}$ being identical. The variable $X_{2}$ must be tested to detect if the constraint can be solved:

$* X_{2}$ is an integer: if $X_{2}=$ Bvalue $_{2}$ then the pair $\left(X_{0}\right.$, Bvalue $\left._{0}\right)$ is enqueued or else the constraint is already solved (Solved).

* $X_{2}$ is a boolean variable: the constraint still suspends (Suspend).

Each constraint $(X, B v a l u e)$ in the queue will be activated and can have one of the following issues:

- Reduce: the boolean variable $X$ is set to the integer Bvalue,

- Check Ok: $X$ already equals Bvalue,

- Fail: $X$ is an integer different from Bvalue.

When a constraint $(X$, Bvalue) has Reduce as issue, the propagation reconsider all constraints depending on $X$. Each such constraint will be enqueued in order to be activated (and taken into account by the above cases) or ignored (only if $n=2$ ) due to:

- Suspend: the other variable of the constraint is not yet ground,

- Solved: the other variable is ground but does not correspond to the "sign" of its literal, i.e. the premisse is false. 


\subsubsection{Optimizations}

Obviously, Check $O k$ corresponds to a useless tell since it neither reduces the variable nor fails. As for clp(FD) we can avoid some of such tells [10. However, in the simpler framework of $c l p(B)$, empirical results show that there is no gain in terms of efficiency. Indeed, a useless tell only consists in a test between two integers and the detection of the possibility to avoid such a tell also involves a test between integers.

The Solved issue also corresponds to a useless work since the constraint is already entailed (see [10]).

Figure 3 makes it possible to estimate the proportion of each issue for some instances of our benchmarks.

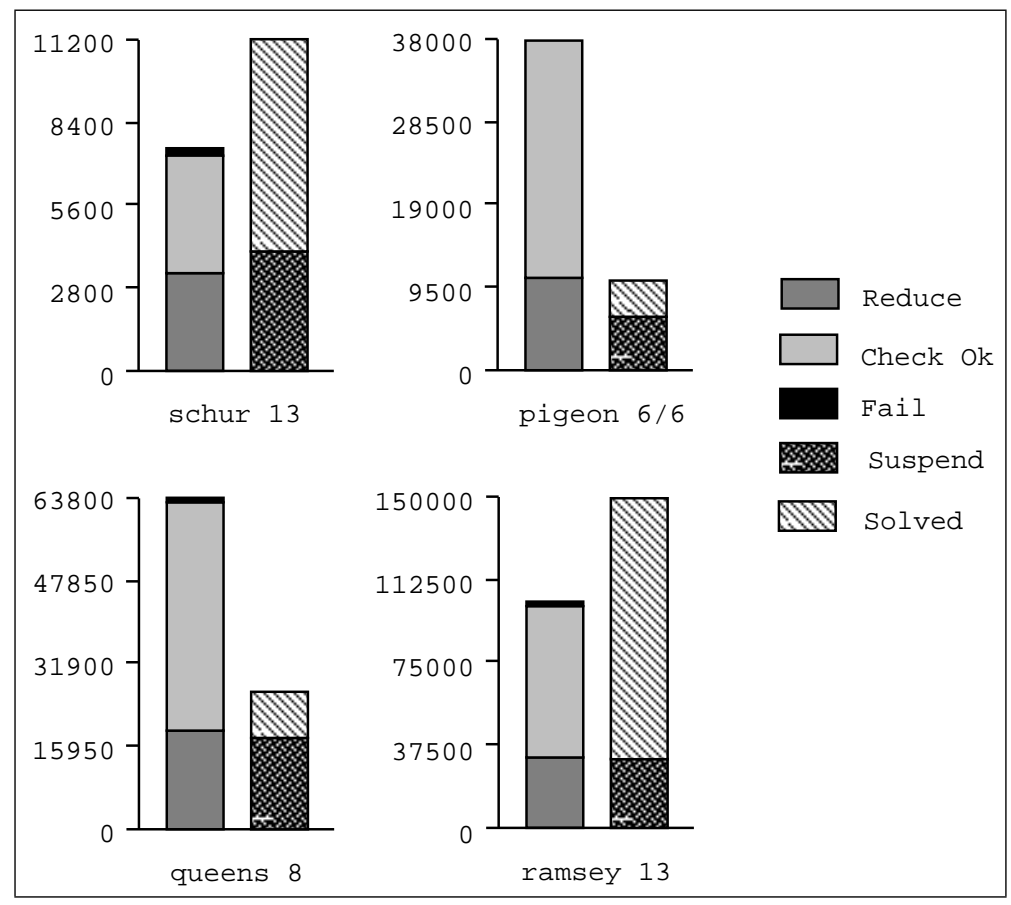

Figure 3: Proportion of each issue of the consistency procedure

\section{Performances of $c l p(B)$}

Table 14 shows the performances of $\mathrm{clp}(\mathrm{B})$ and the corresponding speedup w.r.t. $\operatorname{clp}(\mathrm{B} / \mathrm{FD})$ and all other solvers above presented. The sign "ovflw" means that the program exhausted available memory, and the symbol "?" means that the timing was not available to us.

Basically, $c l p(B)$ is about twice as fast as clp (B/FD) on average. This factor varies only slightly between 1.5 and 2.5 depending on the problem, showing that the two systems perform the same pruning. clp(B) achieves better performances because of its simpler data-structures and internal computations. Therefore, in comparing clp(B) with other solvers, the same arguments apply: we have a general system 
without any specific heuristic versus specialized solvers which usually include very effective heuristics. Nevertheless clp(B) proved to be, on the benchmark problems available, more efficient than all other solvers (except BDDs with the best order for one example), usually by more than one order of magnitude.

\begin{tabular}{||l||r||r|r|r|r|r||}
\hline Program & $\begin{array}{c}\text { clp (B) } \\
\text { Time (s) }\end{array}$ & $\frac{\mathrm{clp} \text { (B/FD) }}{\mathrm{cl} \text { (B) }}$ & $\frac{\text { CHIP }}{\mathrm{clp}(\mathrm{B})}$ & $\frac{\text { Bdd best }}{\mathrm{clp}(\mathrm{B})}$ & $\frac{\text { Enum }}{\mathrm{clp}(\mathrm{B})}$ & $\frac{\mathrm{BCons}}{\mathrm{clp}(\mathrm{B})}$ \\
\hline \hline schur 13 & 0.040 & 2.50 & 20.57 & 27.75 & 20.25 & 1.75 \\
schur 14 & 0.040 & 2.50 & 22.00 & 35.75 & 22.00 & 2.00 \\
schur 30 & 0.100 & 2.50 & 93.70 & ovflw & $?$ & $?$ \\
schur 100 & 0.620 & 1.89 & 322.83 & ovflw & $?$ & $?$ \\
\hline pigeon 6/5 & 0.020 & 2.50 & 15.00 & 3.00 & 2.00 & 6.50 \\
pigeon 6/6 & 0.180 & 2.00 & 10.00 & $\downarrow 1.80$ & 12.72 & 4.88 \\
pigeon 7/6 & 0.110 & 2.81 & 15.45 & 1.00 & 7.63 & 7.90 \\
pigeon 7/7 & 1.390 & 1.91 & 9.67 & $\downarrow 5.56$ & $?$ & 5.20 \\
pigeon 8/7 & 0.790 & 2.81 & 16.12 & $\downarrow 2.54$ & $?$ & 8.63 \\
pigeon 8/8 & 12.290 & 1.97 & 9.58 & $\downarrow 21.18$ & $?$ & 5.49 \\
\hline queens 6 & 0.040 & 1.50 & $?$ & 25.25 & 1.75 & $?$ \\
queens 7 & 0.090 & 1.88 & $?$ & 50.55 & 4.11 & $?$ \\
queens 8 & 0.230 & 2.34 & 19.17 & 233.73 & 6.26 & 7.86 \\
queens 9 & 0.860 & 2.48 & 19.37 & ovflw & 8.02 & 9.01 \\
queens 10 & 3.000 & 2.75 & 22.27 & ovflw & $?$ & 10.90 \\
queens 14 1st & 0.500 & 1.74 & 12.56 & ovflw & $?$ & 6.28 \\
queens 16 1st & 1.510 & 2.17 & 17.47 & ovflw & $?$ & 11.89 \\
queens 18 1st & 4.450 & 2.35 & 20.27 & ovflw & $?$ & $?$ \\
queens 20 1st & 17.130 & 2.51 & 22.93 & ovflw & $?$ & $?$ \\
\hline ramsey 12 1st & 0.130 & 1.46 & 10.53 & ovflw & $?$ & $?$ \\
ramsey 13 1st & 0.690 & 2.17 & 11.13 & ovflw & $?$ & $?$ \\
ramsey 14 1st & 1.060 & 2.28 & 31.30 & ovflw & $?$ & $?$ \\
ramsey 15 1st & 292.220 & 2.39 & 32.10 & ovflw & $?$ & $?$ \\
ramsey 16 1st & 721.640 & 2.52 & 44.17 & ovflw & $?$ & $?$ \\
\hline
\end{tabular}

Table 14: $\operatorname{clp}(\mathrm{B})$ versus all other solvers

\section{Conclusion and perspective}

We have presented several techniques based on local consistency and propagation techniques for solving boolean constraints.

We have formally defined the boolean constraint system by a rule-based operational semantics for the entailment relation which encodes the propagation scheme for boolean constraints and proved its equivalence w.r.t the declarative definition of boolean expressions through truth-tables.

A very simple boolean constraint solver $c l p(B / F D)$, built upon the finite domain constraint logic language $\mathrm{clp}(\mathrm{FD})$, has first been proposed, and we have also proved that this $c l p(B / F D)$ solver really encodes the operational semantics.

The $\operatorname{clp}(\mathrm{B} / \mathrm{FD})$ solver is very efficient, being eight times faster than the CHIP boolean solver on average, and also several times faster than special-purpose standalone boolean solvers of very different nature : resolution methods, BDD-based 
methods, enumerative methods, Operational Research techniques. This proves firstly that the propagation techniques proposed for finite domains are very competitive for booleans and secondly that, among such solvers, the glass-box approach of using a single primitive constraint $X$ in $r$ is very interesting and it makes it possible to encode other domains (such as boolean domains) at a low-level, with better performances than the "black-box". An additional advantage is the complete explicitation of the propagation scheme.

Nevertheless, performances can be improved by simplifying the data-structures used in $\operatorname{clp}(\mathrm{FD})$, which are designed for full finite domain constraints, and specializing them for booleans by explicitly introducing a new type and new instructions for boolean variables. For instance, it is possible to reduce the variable frame representing the domain of a variable and its associated constraints to only two words: one pointing to the chain of constraints to awake when the variable is bound to 0 and the other when it is bound to 1 . Such a solver is very compact and simple; it is based again on the glass-box approach, and uses only a single low-level constraint, more specialized than the $X$ in $r$ construct, into which boolean constraints such as and, or or not are decomposed. This primitive constraint can be implemented in a WAM-based logical engine with a minimal extension : only four new abstract instructions are needed. This surprisingly simple instruction set makes it possible to integrate boolean constraints in any Prolog compiler very easily. Our experience shows that it only requires a few days of implementation work... Moreover, this dedicated solver, called $\operatorname{clp}(\mathrm{B})$, provides a further factor two speedup w.r.t. $\operatorname{clp}(B / F D)$ and is therefore more efficient than all other solvers we have been able to compare with.

It is worth noticing that in clp(FD) the only heuristic available for labeling is the classical "first-fail" based on the size of the domains which is obviously useless for boolean constraints. Some more flexible primitives (e.g. number of constraints on $X$, number of constraints using $X$ ) would be necessary in order to express, at the language level, some complex labeling heuristics [31, 221. Such a labeling however requires complex entailment and disentailment detection for constraint, that we are currently investigating [9]. 


\section{References}

[1] H. Aït-Kaci. Warren's Abstract Machine, A Tutorial Reconstruction. Logic Programming Series, MIT Press, Cambridge, MA, 1991.

[2] F. Benhamou. Boolean Algortihms in PrologIII. In Constraint Logic Programming: Selected Research, A. Colmerauer and F. Benhamou (Eds.). The MIT Press, 1993.

[3] H. Bennaceur and G. Plateau. FASTLI: An Exact Algorithm for the Constraint Satisfaction Problem: Application to Logical Inference. Research Report, LIPN, Universite Paris-Nord, Paris, France, 1991.

[4] H. Bennaceur and G. Plateau. Logical Inference Problem in Variables 0/1. in IFORS 93 Conference, Lisboa, Portugal, 1993.

[5] BNR-Prolog User's Manual. Bell Northern Research. Ottawa, Canada, 1988.

[6] A. Bockmayr. Logic Programming with Pseudo-Boolean Constraints. Research report MPI-I-91-227, Max Planck Institut, Saarbrucken, Germany, 1991.

[7] R.E. Bryant, Graph Based Algorithms for Boolean Fonction Manipulation. IEEE Transactions on computers, no. 35 (8), 1986, pp 677-691.

[8] W. Büttner and H. Simonis. Embedding Boolean Expressions into Logic Programming. Journal of Symbolic Computation, no. 4 (1987), pp 191-205.

[9] B. Carlsson, M. Carlsson, D. Diaz Entailment of Finite Domain Constraints. draft, 1993.

[10] P. Codognet and D. Diaz. Compiling Constraint in clp(FD). draft, 1993.

[11] A. Colmerauer. An introduction to PrologIII. Communications of the ACM, no. 28 (4), 1990, pp 412-418.

[12] M. M. Corsini and A. Rauzy. $\operatorname{CLP}(\mathcal{B})$ : Do it yourself. In GULP'93, Italian Conference on Logic Programming, Gizzeria Lido, Italy, 1993.

[13] D. Diaz and P. Codognet. A Minimal Extension of the WAM for clp(FD). In 10th International Conference on Logic Programming, Budapest, Hungary, The MIT Press 1993.

[14] G. Dore and P. Codognet. A Prototype Compiler for Prolog with Boolean Constraints. In GULP'93, Italian Conference on Logic Programming, Gizzeria Lido, Italy, 1993.

[15] G. Gallo, G. Urbani, Algorithms for Testing the Satisfiability of Propositional Formulae. Journal of Logic Programming, no. 7 (1989), pp 45-61.

[16] R. M. Haralick and G. L. Elliot. Increasing Tree Search Efficiency for Constraint Satisfaction Problems. Artificial Intelligence 14 (1980), pp 263-313

[17] J. N. Hooker and C. Fedjki. Branch-and-Cut Solution of Inference Problems in Propositional Logic. Research Report, Carnegie-Mellon University Pittsurh, Pennsylvania, 1987. 
[18] J. Jaffar and J-L. Lassez. Constraint Logic Programming. In Principles Of Programming Languages, Munich, Germany, January 1987.

[19] A. K. Mackworth. Consistency in Networks of Relations. Artificial Intelligence 8 (1977), pp 99-118.

[20] U. Martin, T. Nipkow, Boolean Unification - The story so far. Journal of Symbolic Computation, no. 7 (1989), pp 191-205.

[21] J-L. Massat. Using Local Consistency Techniques to Solve Boolean Constraints. In Constraint Logic Programming: Selected Research, A. Colmerauer and F. Benhamou (Eds.). The MIT Press, 1993.

[22] U. Montanari. Networks of constraints: Fundamental properties and application to picture processing. In Information Science, 7 (1974).

[23] B. A. Nadel. Constraint Satisfaction Algorithms. Computational Intelligence 5 (1989), pp 188-224.

[24] A. Rauzy. L'Evaluation Sémantique en Calcul Propositionnel. PhD thesis, University of Aix-Marseille II, Marseille, France, January 1989.

[25] A. Rauzy. Adia. Technical report, LaBRI, Université Bordeaux I, 1991.

[26] A. Rauzy. Using Enumerative Methods for Boolean Unification. In Constraint Logic Programming: Selected Research, A. Colmerauer and F. Benhamou (Eds.). The MIT Press, 1993.

[27] A. Rauzy. Some Practical Results on the SAT Problem, Draft, 1993.

[28] V. Saraswat. The Category of Constraint Systems is Cartesian-Closed. In Logic In Computer Science, IEEE Press 1992.

[29] D. S. Scott Domains for Denotational Semantics. In ICALP'82, International Colloquium on Automata Languages and Programmaing, 1982.

[30] H. Simonis, M. Dincbas, "Propositional Calculus Problems in CHIP", ECRC, Technical Report TR-LP-48, 1990.

[31] P. Van Hentenryck. Constraint Satisfaction in Logic Programming. Logic Programming Series, The MIT Press, Cambridge, MA, 1989.

[32] P. Van Hentenryck, V. Saraswat and Y. Deville. Constraint Processing in cc(FD). Draft, 1991.

[33] P. Van Hentenryck, H. Simonis and M. Dincbas. Constraint Satisfaction Using Constraint Logic Programming. Artificial Intelligence no 58, pp 113-159, 1992.

[34] P. Van Hentenryck, Y. Deville and C-M. Teng. A Generic Arc-Consistency Algorithm and its Specializations. Artificial Intelligence 57 (1992), pp 291321.

[35] D. H. D. Warren. An Abstract Prolog Instruction Set. Technical Report 309, SRI International, Oct. 1983. 